\title{
Physical Health and Maternal-Fetal Attachment among Women: Planned Versus Unplanned Pregnancy
}

\author{
Sedigheh Pakseresht ${ }^{1}$, Pegah Rasekh $^{2 *}$, Ehsan Kazemnejad Leili ${ }^{2}$
}

\begin{abstract}
Objectives: The present study aimed at comparing the physical health and maternal-fetal attachment in the women with planned and unplanned pregnancies.

Materials and Methods: This was a descriptive-comparative study including 300 pregnant women (165 planned and 135 unplanned pregnancies) with mean age of $29.8 \pm 5.8$ (age range of 16-50 years old) who had referred to the prenatal clinic of a hospital from September to October, 2016. The maternal-fetal attachment was analyzed using frequency distribution of attachment scores which did not follow the normal distribution of the subscale totally and specifically. Therefore, to compare the scores of the 2 groups, nonparametric tests were used. To calculate the percentage of physical health of the groups, proprietary test was used.

Results: Unplanned pregnancy was assumed as a predictor of physical health because it made women susceptible to health complications (poor health) 5.42 times more than other variables. In 2 groups of the women with planned and unplanned pregnancies, the attachment score was significantly different between the planned and unplanned pregnancy groups.

Conclusions: Unplanned pregnancy makes less maternal-fetal attachment and causes insufficient care as well as insufficient nutrition during pregnancy which in turn leads to unhealthy status in mother and child. Hence, to tackle this problem, reproductive health counseling, education, encouragement and problem-solving in various fields including psychological support of mothers in prenatal care and acceptance of parental role are recommended.

Keywords: Unplanned pregnancy, Physical health, Maternal-fetal attachment
\end{abstract}

\section{Introduction}

Planned pregnancy is an important indicator of improving the health status of mothers and newborns, as today unplanned pregnancy is a public health indicator (1). There are 2 types of unplanned pregnancies; first, the pregnancy which happens sooner than intended pregnancy time called mistimed pregnancy. And the second type which is unintended pregnancy, when a woman has no decision to have a baby in the future (24). Pregnancy and childbirth are joyful experiences for women (4). This event raises pleasure and satisfaction as well as concerns about accepting pregnancy and changes in family and social roles (5). In planned pregnancy, the couple feels stronger; however, women need to get ready for a healthy pregnancy, childbirth, and childcare. On the contrary, unplanned and forced pregnancy makes mothers fearful and anxious $(5,6)$. During this period, mothers may face high risk factors (7) including delayed or no referral to health care centers for receiving pregnancy health care, lack of self-care behaviors (taking no folic acid and iron tablets, no tetanus injection), and increased risk of medical complications and surgery (anemia and increased risk of preeclampsia) (8).
Unplanned pregnancy and its consequences is a global problem which affects women, children, as well as families, and the society $(9,10)$. It often leads to induced abortions and preterm labor, and sometimes its complications can cause mother's death (11).

In developing countries, $20 \%-40 \%$ of births are unplanned or mistimed, posing problems for families and jeopardizing the health of millions of women and children, which lead to an estimated number of 50 million induced abortions(12). In the United States, $45 \%$ of the pregnancies are unplanned, from which $85 \%$ belong to the women who use no contraceptive methods. Forty-five percent of these women are 15-44 years old from which $22 \%$ choose abortion (13). In Iran, annually 400000 500000 unplanned pregnancies (constituting $40 \%$ of total pregnancies) are reported. In a meta-analysis performed in Iran, the total prevalence of unplanned pregnancy was $30.6 \%$. Despite success in Iran's family planning program, one third of pregnancies are unintended and it is increasing 3.2 times per year, and $75 \%$ of these unintended pregnancies are among the couples using the contraceptive methods (1). The most important causes of this problem can be traced back to failure of contraceptive 
methods, improper use of these methods, unfavorable socio-economic status, no desire to have more child, not being ready for child care, concerns about health status of mother and fetus, lack of ability to continue education due to child care, and poor relationship with spouse or desire to get divorced (14). Emotional and psychological problems during unintended pregnancy are one of the most important worries for mother's mental health. In other words, lack of emotional attachment of mother to her fetus is the term used to describe the emotional bond between mother and fetus which increases during pregnancy and reflects in the feelings, perceptions, and behaviors of mother (15). The emotional attachment motivates mothers to acquire motherhood competence and satisfaction for this role after childbirth. Increased attachment makes mothers more interested in healthy behaviors such as smoking and alcohol abstinence during pregnancy, proper nutrition and participation in childbirth education classes (16). The recent researches yield the fact that the maternal-fetal attachment is an intimate and longlasting relationship between mother and child which is satisfying for both of them and facilitates their interaction (17). It is also vital for the survival and growth of the child and its effects are appreciable throughout his/her whole life (18). Many studies have shown that attachment to the fetus is closely related to the emotional conflicts of mother, mother-child relationship, relationship with others, planned or unplanned pregnancy and also tending to have an abortion. The results of a study revealed that the mother-fetal attachment score in the women with planned pregnancies was $23.4 \%$, which is significantly higher $(83.5 \%)$ than that of the women with unplanned pregnancies (16).

Motherhood is a critical period and pregnancy is the best time to help prepare for and cope with motherhood role. Preparing pregnant women to accept this role is the responsibility of a midwife. Moreover, the majority of prenatal care in Iran includes physical care of pregnant women, but little attention is paid to psychological needs of them particularly maternal-fetal attachment; thus pregnancy care is a good opportunity to evaluate maternal-fetal attachment. Furthermore, midwives can have the closest relationship with the pregnant women during pregnancy. They can be efficiently helpful due to their role in reproductive health counseling, educating, encouraging and problem-solving in various ways including psychological support in prenatal care and acceptance of parental role.

Regarding the positive relationship between pregnant women's behavioral health, and their physical health status, the high rate of unplanned pregnancies in Iran which in turn affects the women's health, and the remarkable effect of maternal-fetal attachment on child's psychosocial future,-this study was conducted to examine and compare the physical health and this type of attachment in the women with planned and unplanned pregnancies.

\section{Materials and Methods}

This descriptive-comparative study compared physical health and maternal-fetal attachment in 2 groups of women with planned and unplanned pregnancies, who had referred to a prenatal clinic from September to October 2016. According to the study samples of Karacam et al (8), using a 2 -tailed test with $80 \%$ power test and $0.5 \%$ significance level, 78 persons were included in 2 groups.

Considering the number of variables in each group, 165 ones were included in the planned and 135 ones in the unplanned pregnancy group, who were enrolled in the study and asked to sign an informed consent form. Inclusion criteria were as: gradual availability method, Iranian nationality, singleton pregnancy, gestational age from 32 to 42 weeks (based on first day of the last reliable menstruation with first trimester sonography), and no previous history of severe mental disorders over the last year (according to their statement).

Data collection tools included a researcher-made questionnaire on physical health of pregnancy period and Cranley's 24-item maternal-fetal attachment scale (MFAS) (19). The former was developed based on the findings of a conducted research, national standard guidelines for pregnancy care and relevant literature with four domains of personal, social, and fertility characteristics (16 questions), predisposing factors of high risk pregnancy (16 questions), history of gravidity and parity (16 questions), the results of the tests (Hemoglobin concentration [Hb] $<11 \mathrm{mg} / \mathrm{dL}$ at first and second trimesters, fasting blood sugar $[\mathrm{FBS}] \leq 95$ at first trimester and oral glucose tolerance test [OGTT] [fasting blood glucose less than 95, 1 hour less than 180 and 2 hours less than 150] at first and second trimesters) (20) and nutrition during pregnancy (milk and dairy products, fruit and vegetables, meat and eggs, cereals and iron and folic acid supplements) (5 items). The daily intake of the followings was considered inadequate; grains (less than half a cup of raw beans or one cup of cooked beans), milk or dairy products (less than 3-4 unit/ day, per portion $=$ a glass of milk or a cup of yogurt or 45-60 g of low-fat cheese per portion $=2$ matchbox-size portions) and fruit and vegetables (less than 3-4 servings of different fruits and less than 4-5 servings of vegetables per day).

In order to examine the physical health in the current pregnancy, the patients were firstly asked about the presence of any medical problem such as preeclampsia, gestational diabetes, and renal disease during the current pregnancy. The frequency of any present problem was then compared between both groups with planned and unplanned pregnancies. To determine the highrisk or low-risk pregnancy, the number of predisposing factors for high-risk pregnancy (pregnancy under 18 or over 35 years old, less than 3 years interval from the last childbirth, fifth and higher parity, pregnancy with pills, injections and Intrauterine device=IUD/Tubal ligation $=\mathrm{TL}$, breastfeeding during pregnancy, smoking 
or using other tobacco products, alcohol usage, previous gravidity and parity including: preeclampsia, gestational diabetes, congenital deformities, stillbirths, preterm and late delivery) was considered using qualitative discrete method.

Thelatter, the standardCranley's 24-item MFAS examines mother's behavior toward her fetus on 5 dimensions; accepting the role of motherhood (4 items), interaction with the fetus ( 5 items), attributing characteristics to the fetus (6 items), distinguishing between self and fetus (4 items), and devotion (5 items). The items were graded from 1 to 5 (5: Definitely yes, 4: Yes, 3: I'm not sure 2: No 1: Definitely no). Only in the 22nd item, the scoring was vice versa (1: Definitely yes, 2: Yes, 3: I'm not sure, 4: No, 5: Definitely no). Points were calculated between 24 and 120. Higher scores showed more maternal-fetal attachment. The questionnaires were completed by the researcher through interview. To evaluate the reliability based on a pilot study on 20 pregnant women (10 planned and 10 unplanned pregnancies), Cronbach a coefficient was obtained. It was measured $87 \%$ for maternal-fetal attachment questionnaire and $85 \%$ for physical health questionnaire which indicated the appropriate reliability of these tools.

In order to analyze the data, SPSS software (version 20.0) was used. Maternal-fetal attachment was evaluated using frequency distribution of attachment scores which did not follow the normal distribution of the domains totally and specifically ( $P>0.05$ based on Kolmogorov-Smirnov). Therefore, to compare the scores of the 2 groups, a nonparametric test (Kolmogorov-Smirnov) was used. Chisquare, Mann-Whitney, Kruskal-Wallis, Fisher exact test, and $U$ test were used to compare these scores in 2 groups of the women with planned and unplanned pregnancies. To calculate the risk of physical health problems and reduction of maternal-fetal attachment, regression analysis was employed.

\section{Results}

Of 3348 pregnant women referred to the prenatal clinic with an average of 60 referrals per day, 300 pregnant women were eligible with mean age of $29.8 \pm 5.8$ (age range of 16-50 years old). Most of them (57.3\%) were in age range of 25 to 35 years old. Moreover, $80.7 \%$ of pregnant women and $83.27 \%$ of husbands had high school diploma or higher education, $91.7 \%$ of women were housewives and $51 \%$ of them lived in a renting house, $38 \%$ of pregnant women had their first pregnancy and $66.3 \%$ of them used withdrawal method.

The results of chi-square test revealed that pregnancy complications (preeclampsia, diabetes, blood pressure, FBS disorder, kidney and heart disease) significantly increased with age $(P<0.001)$, gravidity $(P=0.007)$, and parity $(P<0.001)$.

Furthermore, there was a statistically significant correlation between medial complication in the present pregnancy and predisposing factors such as pregnancy $>35$ years $(P=0.008)$, interval from the last childbirth $<3$ years $(P=0.051)$, breastfeeding during pregnancy $(P=0.026)$, history of preeclampsia/eclampsia in previous pregnancies $(P<0.001)$, and a history of preterm birth $(P=0.011)$. Pregnant women with these features were more likely to develop physical health problems than others. Additionally, medical complications associated with abnormal $\mathrm{Hb}$ in the first and third trimesters $(\mathrm{Hb}$ $<11 \mathrm{~mL} / \mathrm{dL}$ ) and fasting blood glucose in first trimester (FBS $\geq 95$ ) and abnormal Oral Glucose Tolerance Test (OGTT; fasting $>95 \mathrm{~mL} / \mathrm{dL}$, more than 1 hour $>180 \mathrm{~mL} /$ $\mathrm{dL}$ more than 2 hours $>155 \mathrm{~mL} / \mathrm{dL})$ in second trimester were significantly increased $(P<0.001)$.

There was also a statistically significant relationship between the medical complications of the current pregnancy and a well-balanced diet. In other words, women having insufficient nutrition were more exposed to medical complications than the others $(P<0.001)$. As shown in Figure 1, the results showed some complications in the women with unplanned pregnancy $(33.3 \%)$ which were significantly $(P<0.001)$ higher $(9.7 \%)$ than those of the women with planned pregnancy.

Our results showed preeclampsia (7.04\%) and gestational diabetes $(8.9 \%)$ in the women with unplanned pregnancy. Planned pregnancy was also accompanied with preeclampsia (2.4\%) and gestational diabetes (0.6\%). Adjusted backward logistic regression was applied to inspect the relationship between physical health status of planned and unplanned pregnancies and control for individual, social and fertility variables. Unplanned pregnancy variable was assumed as a predictor of physical health because it makes women susceptible to health complications (poor health) 5.42 times more (odds ratio $[\mathrm{OR}]=5.42$, 95\% CI; 1.5-19.4). Among other variables, spouse education level $(P=0.04)$, history of preeclampsia $(P<0.001), \mathrm{Hb}$ at first trimester $(P=0.002), \mathrm{Hb}$ at second trimester $(P=0.003)$, impaired fasting blood glucose (FBS $\geq 95)(P<0.001)$, and insufficient dairy consumption (less than 3-4 portions per day $=$ one glass of milk or yogurt

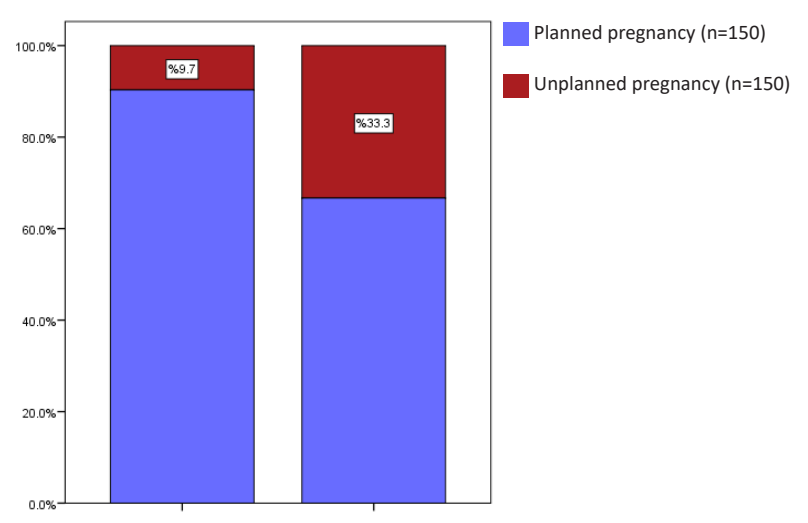

Figure 1. Distribution of Physical Complaints During Pregnancy by Planned and Unplanned Pregnancy. 
Table 1. Logistic Regression of Planned and Unplanned Pregnancies Based on Physical Health Status (Adjusted Model)

\begin{tabular}{lcccccc}
\hline \multirow{2}{*}{ Variables } & \multirow{2}{*}{ B } & \multirow{2}{*}{ SE } & \multirow{2}{*}{ OR } & \multicolumn{2}{c}{$95 \% \mathrm{Cl}$} \\
\cline { 5 - 8 } & & & & & Lower & Upper \\
\hline Type of pregnancy & 1.690 & 0.652 & 0.01 & 5.419 & 1.51 & 19.451 \\
Education level of spouse & 0.462 & 0.225 & 0.04 & 1.586 & 1.022 & 2.464 \\
Preeclampsia in prior pregnancy & 4.364 & 1.197 & 0.0001 & 78.585 & 7.526 & 820.61 \\
Hb (<11) at first trimester & 3.220 & 1.050 & 0.002 & 25.036 & 3.197 & 196.086 \\
Hb (<11) at 2nd trimester & 2.312 & 0.786 & 0.003 & 10.095 & 2.164 & 47.101 \\
Non-fasting blood sugar (FBS >95) & 3.335 & 0.824 & 0.0001 & 28.075 & 5.588 & 141.045 \\
Insufficient intake of dairy products & 1.680 & 0.600 & 0.005 & 5.367 & 1.655 & 17.404 \\
Constant & -17.538 & 2.909 & - & - & - & - \\
\hline
\end{tabular}

Abbreviation: OR, Odds Ratio.

Significant at $P$ value $<0.05$.

45-60 g of low-fat cheese per portion $=2$ match box-size portions) $(P=0.005)$ were predictors of physical health hazards during this critical period (Table 1$)$.

Comparison of the MFAS in all subscales (differentiation of self, interaction with the fetus, attributing characteristics and intentions, self-sacrifice, and role taking) in 2 groups of women based on chi-square test illustrated that the maternal-fetal attachment scores in all subscales and in both groups were statistically significant except for "selfsacrifice" subscale $(P<0.001)$. On the other hand, in the pregnant women, maternal self-sacrifice was not affected by the type of pregnancy (Table 2). Overall, the total score of maternal-fetal attachment of planned pregnancy was higher than that of unplanned pregnancy $(P<0.001)$ (Figure 2).

The results also illustrated that the maternal-fetal attachment was lowered with mother's age over 35 years $(P<0.001)$, school education $(P<0.001)$, less than three years interval from the last childbirth $(P<0.001)$, fifth and higher parity $(P=0.02)$, pregnancy with the failure of contraceptive methods (pills or injections) $(P=0.01)$, and breastfeeding during pregnancy $(P=0.04)$ which

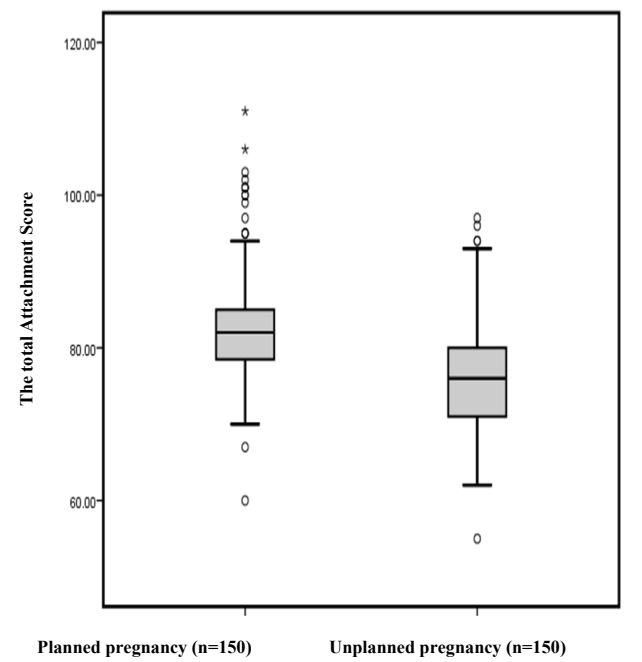

Figure 2. Comparison of the Total Attachment Score Between Planned and Unplanned Pregnancies. was statistically significant. However, the history of preeclampsia, gestational diabetes, congenital deformities, stillbirths and premature and late labor were not significantly related to the maternal-fetal attachment $(P>0.05)$. Moreover, the results indicated a significant relationship between lower attachment score and consumption of meat and eggs $(P<0.001)$, milk or dairy products $(P<0.001)$, fruits and vegetables $(P<0.001)$ and supplements (iron and folic acid) $(P=0.04)$.

Studying the correlation between quantitative variables and maternal-fetal attachment based on Spearman correlation coefficient represented an inverse significant correlation between the number of pregnancies ( $\mathrm{r}=$ -236, $P<0.001)$, parity $(\mathrm{r}=-250, P<0.001)$, maternal age $(\mathrm{r}=-300, P<0.001)$ and a decrease in the number of predisposing factors of high-risk pregnancies ( $\mathrm{r}$ $=-371, P<0.001) \quad($ Table 2). Logistic regression was used to study the relationship between maternal-fetal attachment of planned and unplanned pregnancy variables and controlling individual, social and fertility variables. In this model, the attachment score was divided into 2 categories: below mean (lower attachment) and above mean (higher attachment). The data based on backward logistic regression with probability of inclusion and exclusion of variables from the model $(0.05,0.1$, respectively) showed that among all the variables, type of pregnancy was a predictor of attachment to such a degree that in the planned pregnancy, the mean score of maternal-fetal attachment was 4.05 times more than that in the unplanned pregnancy (OR: 4.05, 95\% CI; $10.47-$ 1.566) (Table 3). Finally, the results of the study showed a statistically significant difference between the planned and unplanned pregnancy attachment scores (Table 4).

\section{Discussion}

Unplanned pregnancy can have adverse effects on physical health of mothers during this period bringing about complications. This study found that physical health of women with planned pregnancy was remarkably higher than that of unplanned pregnancy. The results of a study by Simbar et al reported that pregnant women with 
Table 2. Comparison of Attachment Subscales Scores Based on Planned and Unplanned Pregnancies

\begin{tabular}{|c|c|c|c|c|c|}
\hline Subscales & Type of pregnancy & Number & Mean & SD & $P$ \\
\hline \multirow[t]{2}{*}{ Role taking } & Planned & 165 & 82.3 & 1.82 & 0.0001 \\
\hline & Unplanned & 135 & 26.6 & 1.73 & \\
\hline \multirow[t]{2}{*}{ Interaction with the fetus } & Planned & 165 & 87.9 & 2.55 & 0.0001 \\
\hline & Unplanned & 135 & 35.2 & 2.25 & \\
\hline \multirow[t]{2}{*}{ Attributing characteristics and intentions } & Planned & 165 & 75 & 2.04 & 0.0001 \\
\hline & Unplanned & 135 & 29.8 & 1.54 & \\
\hline \multirow[t]{2}{*}{ Differentiation of self } & Planned & 165 & 74.9 & 1.97 & 0.0001 \\
\hline & Unplanned & 135 & 46.95 & 1.92 & \\
\hline \multirow[t]{2}{*}{ Self-sacrifice } & Planned & 165 & 58.89 & 7.52 & 0.21 \\
\hline & Unplanned & 135 & 36.11 & 6.52 & \\
\hline \multirow[t]{2}{*}{ Total score } & Planned & 165 & 84.44 & 1.82 & 0.0001 \\
\hline & Unplanned & 135 & 31.96 & 1.73 & \\
\hline
\end{tabular}

Table 3. Correlation Between Quantitative Variables and Total Score of the Maternal-Fetal Attachment

\begin{tabular}{lcc}
\hline Variables & Correlation Coefficient $^{\mathrm{a}}$ & $\boldsymbol{P}$ \\
\hline Gravid & -0.236 & 0.0001 \\
Age & -0.3 & 0.0001 \\
Parity & -0.25 & 0.0001 \\
Gestational age & 0.005 & 0.917 \\
Number of risk factors of & -0.371 & 0.0001 \\
high-risk pregnancy & &
\end{tabular}

a Spearman's rho.

unplanned pregnancy had a significantly lower physical health, though unlike the present study, no significant differences were observed in medical and surgical problems between the 2 groups (21). This difference might be due to the study of physical health from different aspects. In their study, physical health was examined in terms of prenatal care, behavioral self-care about nutrition, personal hygiene, and high-risk behaviors such as smoking, alcohol consumption and drugs use (21); while in this study, physical health of mothers was evaluated considering the medical complications such as preeclampsia, gestational diabetes, hypertension, FBS, and heart and kidney disease in the current pregnancy. The results of this study showed that the frequency of physical problems was higher during unplanned pregnancy that was in line with the study of Karacam et al (8). Thus, unplanned pregnancy has negative impacts on women's health. It increases the risk of physical problems and reduces positive behaviors such as seeking medical care during pregnancy (8).

Theme-Filha et al realized that the women with unplanned pregnancies had experienced higher medical problems during their previous pregnancy $(\mathrm{OR}=1.21$, $95 \%$ CI: 1.04-1.41) (4); that was in line with this study results. Medical problems in the women with unplanned pregnancy were significantly higher in comparison with the women with planned pregnancy, and unplanned pregnancy was considered as a predictor of physical health which could increase the hazards to physical health up to 5.4 times. Theme-Filha et al also confirmed the effects of personal, social and financial facets on unplanned pregnancy, expressing that various factors (age under 20 [OR $=1.89,95 \%$ CI: 1.68-2.14], education level under 7 years, 3 or more labors, and multiple sex partners) can complicate unplanned pregnancy (4).

Goyal et al disclosed that medical complications in the women younger than 18 and above 35 years old, breastfeeding during pregnancy, gravidity $\geq 4$, history of preeclampsia/eclampsia, congenital deformities and stillbirths, and preterm/post term delivery were more at risk during pregnancy (22). Moreover, Ashraf Ali et

Table 4. Logistic Regression of Maternal-Fetal Attachment Based on Some Demographic Variables in Planned and Unplanned Pregnancies

\begin{tabular}{|c|c|c|c|c|c|c|}
\hline \multirow{2}{*}{ Variables } & \multirow{2}{*}{ B } & \multirow{2}{*}{ SE } & \multirow{2}{*}{$P$} & \multirow{2}{*}{ OR } & \multicolumn{2}{|c|}{$95 \% \mathrm{Cl}$} \\
\hline & & & & & Lower & Upper \\
\hline Planned/unplanned pregnancy & 1.399 & 0.485 & 0.004 & 4.05 & 1.566 & 10.473 \\
\hline Preeclampsia/eclampsia & 3.775 & 1.122 & 0.001 & 43.603 & 4.837 & 393.035 \\
\hline Insufficient/sufficient intake of dairy products & 1.198 & 0.481 & 0.013 & 3.313 & 1.29 & 8.508 \\
\hline
\end{tabular}

Abbreviation: OR, Odds Ratio.

Significant at $P$ value $<0.05$ 
al believed that preeclampsia and anemia were the most common threatening factors of mother's physical health (23).

Considering the relationship between maternal-fetal attachment and planned/unplanned pregnancy, the results showed that type of pregnancy can be a predictor of attachment, and the attachment score in the women with unplanned pregnancies was significantly less than that in the women with planned ones. Similarly, Ossa et al reported that $61.5 \%$ of the women with unplanned pregnancies had significantly less maternal-fetal attachment (16). Ustunsoz et al also concluded that the attachment score in the planned pregnancy was more than that in the other type (24) which was in line with the present study; while Torshizi and Sharifzadeh found that the mean score of maternal-fetal attachment had no significant difference in planned versus unplanned pregnancies (15). This difference may be due to the differences in sample size and cultural and social characteristics of the research population.

Similar to the results of this study, Hurtado (25) and Punamaki et al suggested that the five subscales of maternal-fetal attachment except for devotion could be affected by intentional or non-intentional status. Another research reported that the feeling of motherhood never vanishes especially her devotion toward the fetus which augments as fetus ages and remains throughout the child's life (27). Torshizi and Sharifzadeh studied the relationship of maternal-fetal attachment with social and individual variables and showed that the mean score of attachment based on such variables as gestational age, parity, highrisk or low-risk pregnancy, housing and income status was not significantly different in the 2 groups (15). However, in the present study a significant correlation was observed between gestational age, gravidity, parity and the number of exposing factors of high-risk pregnancy which represents a reverse correlation, though it is directly linked with parent's education level.

Moreover, a significant correlation between lower attachment score and insufficient nutrition (meat, egg, milk and dairy products, fruit and vegetables) and supplements such as iron and folic acid was observed in which the women with lower attachment score were less interested in a healthful diet and consumption of supplements during their pregnancy; though Torshizi and Sharifzadeh indicated that the mean score of maternalfetal attachment in terms of family income and insufficient nutrition had no change. This difference can be attributed to the varying diets, because in this study mothers' diet during pregnancy has been examined based on national guidelines for pregnancy diet in prenatal care (15).

This study revealed that attachment score in the women with diploma and higher education level was more than that in the women with lower education level. On the contrary, Kwon and Bang found that attachment score was not significantly correlated with mother's education
(27); this could be due to differences in cultures and sample sizes, in which mothers with higher education have more awareness and knowledge of pregnancy issues, fetal growth and development which could be a reason for more attachment to fetus.

It is fair to state that samples in this study were the pregnant women who had referred to prenatal clinic to receive usual prenatal care advice during pregnancy and were not trained about the ways to enhance the maternalfetal attachment; this could be a clue for future studies. Moreover, apart from the main variables of planned or unplanned pregnancies, considering demographic and prenatal characteristics as well as nutrition which were proved to be in relation with maternal-fetal attachment in this study, they can be helpful in educational and health planning in order to improve the attachment and health status of mothers and prenatal outcomes in newborns.

A limitation of this study was mental status of pregnant women which could be effective on their answers to questions.

\section{Conclusions}

As shown in this study, unplanned pregnancy makes less maternal-fetal attachment, and insufficient care as well as insufficient nutrition during pregnancy leads to unhealthy statuses in mother and child. Therefore, public health status would be in danger.

\section{Conflict of Interests}

Authors declare that they have no conflict of interests.

\section{Ethical Issues}

This study was approved by Social Determinants of Health Research Center (SDHRC) and Ethics Committee of Guilan University of Medical Sciences (Code of Ethics: 93245, IR.GUMS .REC.1395.142).

\section{Financial Support}

Financial support was obtained from Guilan University of Medical Sciences, Rasht, Iran.

\section{Acknowledgements}

This article was extracted from an approved proposal. The authors wish to thank the staff of prenatal clinic of Al-Zahra hospital, Rasht, and all mothers for their sincere cooperation.

\section{References}

1. Moosazadeh M, Nekoei-Moghadam M, Emrani Z, Amiresmaili M. Prevalence of unwanted pregnancy in Iran: a systematic review and meta-analysis. Int J Health Plann Manage. 2014;29(3):e277-290. doi:10.1002/hpm.2184

2. Yazdkhasti M, Pourreza A, Pirak A, Abdi F. Unintended Pregnancy and Its Adverse Social and Economic Consequences on Health System: A Narrative Review Article. Iran J Public Health. 2015;44(1):12-21.

3. McKeating A, Maguire PJ, Farren M, Daly N, Sheehan SR, 
Turner MJ. The clinical outcomes of unplanned pregnancy in severely obese women. J Matern Fetal Neonatal Med. 2016;29(4):646-650. doi:10.3109/14767058.2015.1015414

4. Theme-Filha MM, Baldisserotto ML, Fraga AC, Ayers S, da Gama SG, Leal MD. Factors associated with unintended pregnancy in Brazil: cross-sectional results from the Birth in Brazil National Survey, 2011/2012. Reprod Health. 2016;13(Suppl 3):118. doi:10.1186/s12978-016-0227-8

5. van Ditzhuijzen JM. Abortion and mental health: A longitudinal study of common mental disorders among women who terminated an unwanted pregnancy. The Netherland: Utrecht University; 2017.

6. Nisand I, Bettahar K. Medical management of unwanted pregnancy in France: modalities and outcomes. The aMaYa study. Eur J Obstet Gynecol Reprod Biol. 2015;184:13-18. doi:10.1016/j.ejogrb.2014.10.025

7. Goossens J, Van Den Branden Y, Van der Sluys L, et al. The prevalence of unplanned pregnancy ending in birth, associated factors, and health outcomes. Hum Reprod. 2016;31(12):2821-2833. doi:10.1093/humrep/dew266

8. Karacam Z, Onel K, Gercek E. Effects of unplanned pregnancy on maternal health in Turkey. Midwifery. 2011;27(2):288-293. doi:10.1016/j.midw.2009.07.006

9. Stewart H, McCall SJ, McPherson C, et al. Effectiveness of peri-abortion counselling in preventing subsequent unplanned pregnancy: a systematic review of randomised controlled trials. J Fam Plann Reprod Health Care. 2016;42(1):59-67. doi:10.1136/jfprhc-2014-101096

10. Worku S. Prevalence of unintended pregnancy and/or child birth and its determinants in Harar town [Thesis]. Ethiopia: Addis Ababa University; 2002.

11. Boden JM, Fergusson DM, Horwood LJ. Outcomes for Children and Families Following Unplanned Pregnancy: Findings from a Longitudinal Birth Cohort. Child Indic Res. 2015;8(2):389-402. doi:10.1007/s12187-014-9241-y

12. Gerdts C, Dobkin L, Foster DG, Schwarz EB. Side Effects, Physical Health Consequences, and Mortality Associated with Abortion and Birth after an Unwanted Pregnancy. Womens Health Issues. 2016;26(1):55-59. doi:10.1016/j. whi.2015.10.001

13. Finer LB, Zolna MR. Unintended pregnancy in the United States: incidence and disparities, 2006. Contraception. 2011;84(5):478-485. doi:10.1016/j. contraception.2011.07.013

14. Mortazavi F, Damghanian M, Mottaghi Z, Shariati M. Women's experiences of unwanted pregnancy. Behbood. 2012;15(6):492-503.

15. Torshizi M, Sharifzadeh G. Maternal -fetal attachment and associated factors in pregnant women referred to Birjand health centers (2012). J Birjand Univ Med Sci.
2013;20(3):279-287.

16. Ossa X, Bustos L, Fernandez L. Prenatal attachment and associated factors during the third trimester of pregnancy in Temuco, Chile. Midwifery. 2012;28(5):e689-696. doi:10.1016/j.midw.2011.08.015

17. Nieto L, Lara MA, Navarrete L. Prenatal Predictors of Maternal Attachment and Their Association with Postpartum Depressive Symptoms in Mexican Women at Risk of Depression. Matern Child Health J. 2017;21(6):12501259. doi:10.1007/s10995-016-2223-6

18. Lee SH, Lee EY. Factors Influencing Maternal-Fetal attachment in High-Risk Pregnancy. Advanced Science and Technology Letters. 2015;104:38-42. doi:10.14257/ astl.2015.104.09

19. Cranley MS. Development of a tool for the measurement of maternal attachment during pregnancy. Nurs Res. 1981;30(5):281-284. doi:10.1097/00006199-19810900000008

20. Cunningham FG, Leveno KJ, Bloom SL, et al. Williams Obstetrics. 24th ed. USA: McGraw-Hill; 2014.

21. Simbar M, Khajehpoor M, Jannesari S, Alavi Majd H. Comparing the health status of women with wanted and unwanted pregnancy. J Gorgan Univ Med Sci. 2012;14(1):113-120.

22. Goyal SG, Shah PT, Deliwala KJ. Role of fetal monitoring in high risk pregnancy by fetal electrocardiogram. Int J Reprod Contracept Obstet Gynecol. 2014;3(4):893-897. doi:10.5455/2320-1770.ijrcog20141204

23. Ashraf Ali M, Babitha MC, Lokeshchandra HC, Sharma KD, Zehra M, Reddy MS. A study of changing trends of maternal mortality at the tertiary care centre, MMC \& RI Mysore, India. Int J Reprod Contracept Obstet Gynecol. 2015;4(1):239-242. doi:10.5455/2320-1770.ijrcog20150243.

24. Ustunsoz A, Guvenc G, Akyuz A, Oflaz F. Comparison of maternal-and paternal-fetal attachment in Turkish couples. Midwifery. 2010;26(2):e1-9. doi:10.1016/j. midw.2009.12.006

25. Hurtado M. A program to improve maternal-fetal attachment among Latina mothers: A grant proposal. USA: California State University, Long Beach; 2015.

26. Punamaki RL, Isosavi S, Qouta SR, Kuittinen S, Diab SY. War trauma and maternal-fetal attachment predicting maternal mental health, infant development, and dyadic interaction in Palestinian families. Attach Hum Dev. 2017;19(5):463-486. doi:10.1080/14616734.2017.1330833

27. Kwon MK, Bang KS. [Relationship of prenatal stress and depression to maternal-fetal attachment and fetal growth]. J Korean Acad Nurs. 2011;41(2):276-283. doi:10.4040/ jkan.2011.41.2.276

(C) 2018 The Author (s); This is an open-access article distributed under the terms of the Creative Commons Attribution License (http://creativecommons.org/licenses/by/4.0), which permits unrestricted use, distribution, and reproduction in any medium, provided the original work is properly cited. 